Disponible en ligne : www.cahiersagricultures.fr

\title{
Monument historique ou système bien vivant ? Les foggaras des oasis du Touat (Algérie) et leur réalimentation en eau par pompage
}

\author{
Salem Idda ${ }^{1,2,3, *}$, Bruno Bonté ${ }^{4}$, Hamidi Mansour ${ }^{5}$, Sid-Ahmed Bellal ${ }^{6}$ et Marcel Kuper ${ }^{3,7}$ \\ ${ }^{1}$ Département de géographie et d'aménagement du territoire, Faculté des sciences de la terre et de l'univers, Université d'Oran 2 Mohamed \\ Ben Ahmed, Oran, Algérie \\ ${ }^{2}$ Département des sciences de la nature et de la vie, Faculté des sciences et de la technologie, Université Ahmed Draia, Adrar, Algérie \\ 3 CIRAD, UMR G-EAU (Gestion de l'Eau, Acteurs, Usages), Montpellier, France \\ 4 IRSTEA, UMR G-EAU (Gestion de l'Eau, Acteurs, Usages), Montpellier, France \\ ${ }^{5}$ Laboratoire géoressources, environnement et risques naturels, Université d'Oran 2 Mohamed Ben Ahmed, Oran, Algérie \\ ${ }^{6}$ Laboratoire espace géographique et aménagement du territoire, Département de géographie et d'aménagement du territoire, Université \\ d'Oran 2 Mohamed Ben Ahmed, Oran, Algérie \\ ${ }^{7}$ IAV Hassan II, Rabat, Maroc
}

\begin{abstract}
Résumé - Durant les cinq dernières décennies, le Sahara algérien a connu plusieurs programmes de développement agricole fondés sur l'exploitation de l'eau souterraine. Les pompages d'eau souterraine effectués dans les nouveaux périmètres de mise en valeur sont souvent montrés du doigt pour avoir fragilisé les systèmes hydrauliques ancestraux des foggaras (ou qanat, galeries drainant la nappe vers une oasis). Pourtant, dans les régions de Touat, Gourara et Tidikelt, les paysanneries continuent à exploiter un certain nombre de foggaras, parfois même alimentées par des eaux souterraines pompées. L'étude de l'oasis de Lahmeur dans la zone de Touat montre les nouvelles stratégies développées par les oasiens face aux transformations dans la zone. À travers une enquête sur cinq foggaras alimentées par un forage, l'objectif est de décrire et d'expliquer les conditions ayant permis de construire ce nouveau dispositif hydraulique hybride, et la capacité des oasiens à faire des ajustements dans ce système irrigué afin de perpétuer son fonctionnement. Cet article vise à contribuer à l'analyse du processus de renouveau de l'agriculture oasienne dans le Sahara algérien. L'étude permet, d'une part, de souligner certaines ruptures observées dans le fonctionnement du système des foggaras, et, d'autre part, de montrer les continuités et les ajustements opérés par les acteurs dans un contexte saharien en forte mutation.
\end{abstract}

Mots clés : oasis / foggara / qanat / forage / Adrar

\begin{abstract}
Historical monument or living system? Integrating pumped groundwater in the foggaras of the oases of the Touat, Algeria. Over the past five decades, several agricultural development programs based on the use of pumped groundwater were carried out in the Algerian Sahara. The increased groundwater use for the new irrigation schemes is often held responsible for the decline of the ancient foggara (or qanat, subterranean channels collecting groundwater diverted to oases) irrigation systems. However, the peasantry of the Touat, Gourara and Tidikelt regions continues to exploit a certain number of foggaras, sometimes even fed by pumped groundwater. The study of the Lahmeur oasis in the Touat region shows the new strategies developed by the oasis peasantry to adapt to recent transformations. Through a survey of five foggaras fed by a borehole, we analyze the conditions that made it possible to build a hybrid irrigation system and we show the capacity of the irrigation community to adapt this irrigation system to perpetuate its operation. This article aims to contribute to the recent debate on the renewal of oasis agriculture in the Algerian Sahara. It highlights some ruptures in the functioning of foggaras system, but also many continuities and adjustments made by the actors in a rapidly changing Saharan context.
\end{abstract}

Keywords: oasis / foggara / qanat / borehole / Adrar

\footnotetext{
* Auteur de correspondance : iddasalem@gmail.com
} 


\section{Introduction}

Le Sahara algérien fait l'objet depuis cinq décennies de programmes de développement agricole qui visent la sécurité alimentaire du pays et l'amélioration des conditions de vie de la population. Ces multiples programmes ont engendré une transformation profonde du système oasien, allant de son organisation sociale à sa configuration spatiale (Bellal et al., 2016). Aujourd'hui, l'orientation qui consiste à multiplier les programmes de développement à travers des périmètres de mise en valeur agricole dans le Sahara continue à occuper une place importante dans les discours et les programmes publics, et cela en dépit des résultats mitigés sur les plans économique, social et environnemental (Otmane et Kouzmine, 2013).

Dans les régions de Touat, Gourara et Tidikelt, qui font partie de la circonscription administrative d'Adrar dans le sudouest de l'Algérie, la mise en place des nouveaux périmètres de mise en valeur, basés sur l'exploitation des eaux souterraines, est accompagnée par un discours sur le déclin des systèmes hydrauliques ancestraux que sont les foggaras. Une foggara est une galerie drainant le toit d'une nappe souterraine et conduisant par gravité l'eau vers des parcelles de cultures (Bisson, 2003). Ce système de captage et de partage de l'eau est retrouvé dans de nombreux pays depuis la Chine jusqu'au Maroc (El Faiz et Ruf, 2010) en passant par la péninsule Arabe et surtout l'Iran où la présence des qanats est attestée depuis plus de 2000 ans (Mostafaeipour, 2010). Dorénavant, ces systèmes hydrauliques cohabitent souvent avec des périmètres irrigués par pompage de l'eau souterraine. Ainsi, dans le Sahara algérien, la nouvelle situation agricole «juxtapose sur courte distance les formes [agricoles] les plus modernes aux plus traditionnelles » (Côte, 2002). L'exploitation de la nappe du Continental intercalaire dans les nouvelles extensions, souvent installées en amont de la zone de captage des foggaras, par des moyens de pompage puissants a conduit à des rabattements considérables du niveau piézométrique. Selon l'Agence nationale des ressources hydrauliques (ANRH), sur les 2000 foggaras inventoriées à Adrar en 2016, 1278 foggaras sont taries du fait des abaissements du niveau de la nappe. Selon la même agence, le reste des foggaras présente un manque d'entretien et le débit total capté par les foggaras à Adrar a ainsi diminué de $3,6 \mathrm{~m}^{3} / \mathrm{s}$ en 1960 à $1,8 \mathrm{~m}^{3} / \mathrm{s}$ en 2011 .

Le discours décliniste portant sur les foggaras résulte d'abord d'une vision moderniste qui condamne les foggaras au nom de la productivité face à des modes de mise en valeur individualisés jugés plus efficients (Bisson, 2003). Cependant, la vision du déclin des foggaras pour des raisons sociales et physiques est ancienne et précède la mise en place des périmètres de mise en valeur: "un peu partout, on note un déclin des débits d'eau, un abandon des jardins, le nombre des 'foggaras mortes', qui ne fonctionnent plus depuis une époque récente, est encore une preuve de la désaffection pour une technique qui avait vivifié le désert »(Papy, 1959). Cette vision a été reprise dans la littérature depuis une vingtaine d'années pour faire le lien causal explicite entre le déclin des foggaras et l'arrivée massive des ouvrages modernes d'exploitation des nappes, à la fois du fait de la concurrence sur la ressource en eau souterraine mais aussi de la facilité d'exploitation de l'eau souterraine réalisée par pompage (Dubost et Moguedet, 1998 ; Lightfoot, 1996). Le problème n'est pas tant le tarissement des foggaras en lui-même, qui constitue une contrainte inhérente et bien documentée de ce dispositif hydraulique (Balland, 1992), que la rapidité avec laquelle les baisses des nappes interviennent laissant peu de temps aux oasiens pour réagir. Il faut enfin évoquer le contexte spécifique de ces régions, où les réponses traditionnelles (ajout de galeries latérales, approfondissement de la galerie mère, construction d'une nouvelle foggara, relocalisation des parcelles plus bas pour pouvoir continuer à profiter de l'écoulement gravitaire) ne paraissent plus adaptées à la situation sociale et économique actuelle (Bendjelid et al., 1999).

Pourtant, les paysanneries de Touat, comme celles de Gourara et Tikidelt, continuent à montrer un «réel dynamisme», qui se manifeste, d'abord et surtout, dans les nouveaux périmètres de mise en valeur agricole en libérant les agriculteurs des «contraintes topographiques» et des «pesanteurs sociales» (Bisson, 2003). Les descendants des harratins (anciens khammès ou métayers au cinquième), catégorie sociale défavorisée possédant un savoir-faire reconnu dans les pratiques agricoles oasiennes et les travaux d'entretien des foggaras (Marouf, 2017), ont saisi l'occasion de l'installation des nouveaux périmètres afin de bénéficier des terres et des subventions de l'État pour équiper de nouveaux jardins et assurer des extensions dans les oasis (Côte, 2002). Toutefois, à côté des nouveaux périmètres, un dynamisme, parfois latent, est présent «jusque dans les vieilles sociétés hydrauliques » (Bisson, 2003). Les paysanneries, habituées à adapter continuellement leurs systèmes hydrauliques, se sont servies des nouvelles opportunités apportées par la «modernité» pour réinventer les foggaras. Par exemple, elles ont profité de l'arrivée des motopompes pour rénover la foggara: de nouvelles parcelles sont installées en amont et au-dessus des foggaras, ou alors les foggaras sont alimentées à partir d'eaux pompées (Bisson, 1992).

La recherche entreprise sur l'oasis de Lahmeur vise à montrer, à travers une étude de cas, comment une société oasienne a pu retrouver et adapter un dispositif hydraulique «ancien » en adoptant un mode de fonctionnement qui intègre à la fois les opportunités du monde moderne et les pratiques techniques et sociales traditionnelles, ce qui a permis au système oasien de perdurer. Nous émettons l'hypothèse que le fonctionnement actuel des foggaras de Lahmeur garde des continuités et des traces importantes héritées de l'ancien système. Nous explorons ici une hypothèse formulée par Bisson (1992) qui postulait que la combinaison de la technique foggarienne traditionnelle avec des moyens d'exhaure modernes pouvait donner un nouvel élan aux foggaras. Nous supposons également que le nouveau système est une adaptation des communautés aux transformations dans la zone par des ajustements et le maintien de l'action collective, caractéristique principale de l'ancien système, et non pas un nouveau système coupé des expériences accumulées durant des siècles.

\section{Méthodes}

L'oasis de Lahmeur se situe dans le sud-ouest de l'Algérie dans la zone de Touat à $50 \mathrm{~km}$ au sud de la ville d'Adrar (Fig. 1). La zone est caractérisée par son climat hyperaride où les ressources en eau sont uniquement souterraines. 


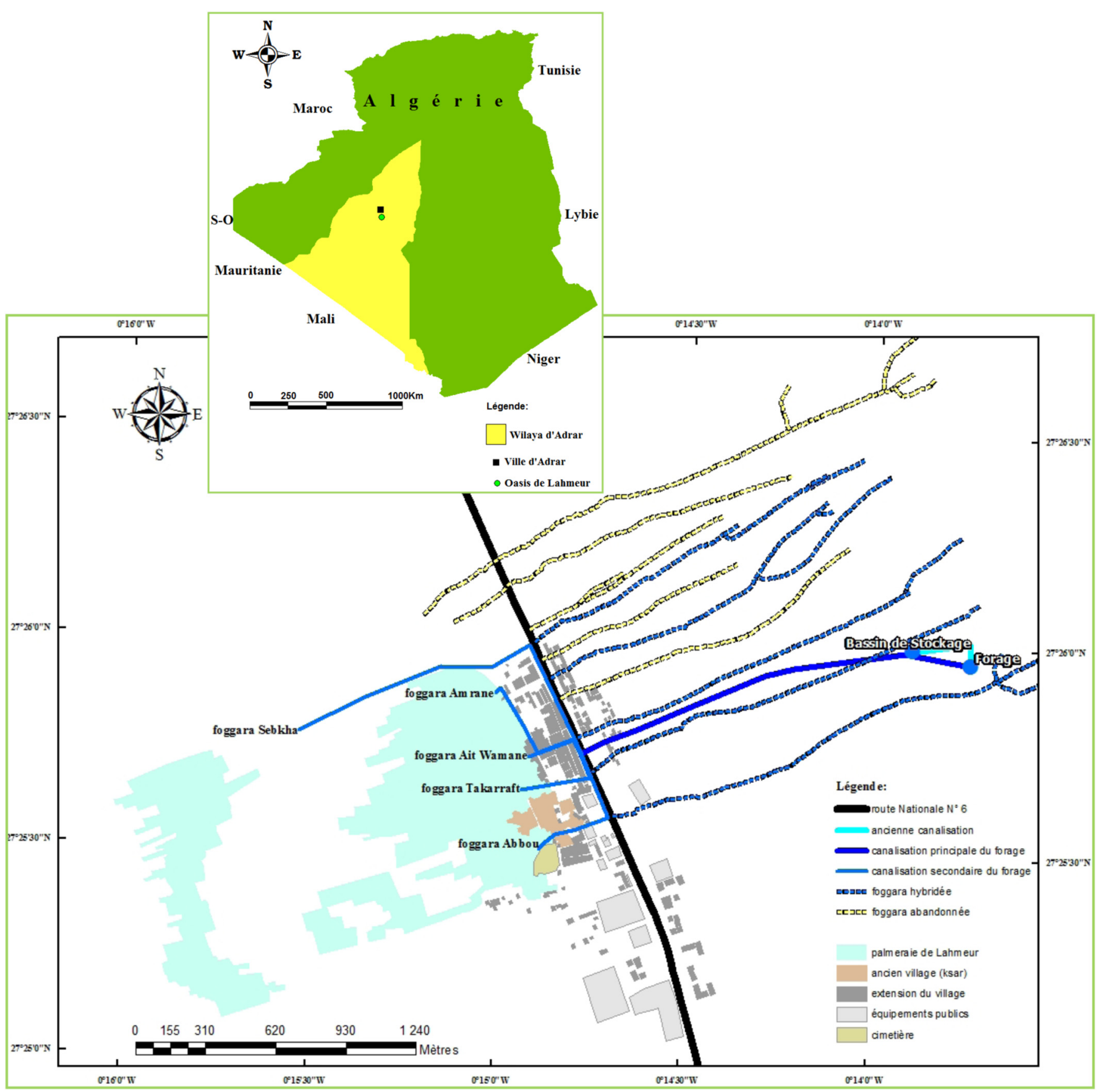

Fig. 1. Situation et organisation spatiale de l'oasis de Lahmeur (source: Google-Earth et enquête de terrain 2017).

Fig. 1. Location and spatial organization of the Lahmeur oasis (source: Google-Earth and field survey 2017).

L'agriculture est pratiquée en trois étages: palmier dattier, arbres fruitiers, céréales et légumes, ce qui permet d'assurer le microclimat nécessaire aux cultures fragiles, les légumes en particulier (tomates, aubergines, courgettes...). Lahmeur est le chef-lieu de la commune de Tammest qui regroupe 11 oasis. Sa palmeraie est d'une superficie d'environ 200 ha irrigués par les eaux captées par un forage mis en place en 1992 et réparties sur cinq foggaras taries. Avec une profondeur de $150 \mathrm{~m}$ et un niveau statique de $30 \mathrm{~m}$, le forage délivre un débit de $26 \mathrm{~L} / \mathrm{s}$.

Le choix de cette oasis a été fait après une enquête préliminaire réalisée en 2016 sur les oasis d'Adrar. Nous avons visité et interviewé des personnes ressources dans plus de 20 oasis réparties sur les régions de Touat, Gourara et Tidikelt. L'oasis de Lahmeur a été l'une des premières à avoir pu installer un forage pour alimenter le système de foggaras, au moment même où le programme de «renforcement» des foggaras par des forages rencontrait un refus généralisé dans les autres oasis. Enfin, c'est le plus ancien forage de renforcement que l'on ait trouvé encore en fonctionnement, 25 ans après son installation. Sur le terrain, nous avons identifié une dizaine de forages en fonctionnement et environ 20 autres en cours de réalisation. La réhabilitation des foggaras faisait 
partie des programmes nationaux de développement, comme le Programme national de développement agricole (PNDA) (Daoudi et al., 2015). Aujourd'hui, ces opérations sont financées par les programmes sectoriels des services des ressources en eau et de l'agriculture d'Adrar.

Le présent article résulte surtout d'une $2^{\mathrm{e}}$ phase de terrain réalisée en 2017. Nous avons commencé par interviewer trois personnes ressources, le président de l'association des foggaras, son adjoint et un retraité, ancien délégué de la Direction des services agricoles de la commune de Tammest au moment de l'installation du forage. Au cours de l'enquête, nous avons visité 47 parcelles représentant environ 40 ha des 200 ha alimentés par les cinq foggaras. Nous avons relevé des données sur l'organisation spatiale, l'infrastructure hydraulique et l'organisation sociale et institutionnelle du nouveau système de captage et de distribution de l'eau. Nous avons analysé les documents écrits de l'association des foggaras: listes des propriétaires dans chaque foggara, documents de création de l'association, règlement intérieur de gestion. Les résultats ont été confrontés aux données recueillies par des entretiens semi-directifs auprès de 15 ayants droit à l'eau du forage. Ces derniers ont été choisis en concertation avec les trois personnes ressources pour couvrir les différents cas d'accès à l'eau: neuf anciens propriétaires (dont quatre descendants des anciens khammès) et six nouveaux propriétaires ayant eu des droits d'eau après la mise en place du forage. Nous avons rencontré des difficultés pour identifier les différentes catégories sociales aujourd'hui actives dans l'oasis et disposant de droits d'eau, du fait des reconfigurations sociales et des susceptibilités que l'ancienne appartenance servile continue à poser dans le contexte oasien. Ce déficit d'information a été complété par l'analyse des documents déjà cités et des discussions avec certaines personnes ressources dans les oasis avoisinantes.

\section{Résultats}

\subsection{Périmètres de mise en valeur agricole et réhabilitation des foggaras : des objectifs difficilement conciliables}

Adrar était l'une des principales wilayas concernées par les programmes de développement agricole dans le Sahara algérien. L'opération «tomate d'Adrar» pour la mise en place «d'une culture extra-primeur et ... d'une filière intégrée (tomate/conserverie/marché extérieur)" fut, en effet, initiée dès le début des années 1970 (Sahli, 1997). Durant les années 1980 , des périmètres de «petite» et de «grande» mise en valeur agricole ont été installés, dans le cadre de l'application de la loi de l'Accession à la propriété foncière agricole (APFA), et durant les années 2000 par le Plan national de développement agricole (PNDA). La petite mise en valeur agricole concerne des collectifs de paysans et de jeunes chômeurs locaux pour des exploitations allant de 2 à 10 ha, et la grande mise en valeur concerne des surfaces allant de 100 à 5000 ha au profit de notables locaux ou d'investisseurs venus d'ailleurs. Cependant, en 2005, moins de 5\% des superficies attribuées à la grande mise en valeur ont été réellement mises en culture, montrant un grand décalage entre les ambitions et les réalisations de ces programmes (Otmane et Kouzmine, 2013).
À Lahmeur, un nouveau périmètre avait été installé à $2 \mathrm{~km}$ en amont de la zone de captage des foggaras. Les bénéficiaires appartenaient à l'oasis voisine de Tittaf. Les superficies des terrains exploités étaient de 1 à 4 ha pour la petite mise en valeur agricole et de 100 à 237 ha pour la grande mise en valeur agricole. À Lahmeur, deux des trois personnes ressources ont évoqué l'année 1989 pour désigner la date de début de tarissement des cinq foggaras de l'oasis. Les images satellites (Google-Earth) montrent que cette année correspond au début de l'exploitation du nouveau périmètre de mise en valeur agricole. Deux autres périmètres de grande mise en valeur agricole, de surface plus importante, ont été installés à 12 et $15 \mathrm{~km}$, respectivement au nord-est et au sud-est des foggaras. Les images satellites montrent qu'ils ne sont entrés en exploitation qu'à partir de 1991.

Dans la wilaya d'Adrar, l'installation des nouveaux périmètres a eu un double effet. D'abord, l'impact a été ressenti en termes de baisse dans les débits des foggaras, en particulier au sein des exploitations proches des nouveaux périmètres, ce qui a provoqué des oppositions à l'installation de certains périmètres en amont des foggaras. Ensuite, les agriculteurs les plus dynamiques et qualifiés, en particulier les anciens khammès qui ont vu dans ces périmètres une voie d'émancipation par l'accès à l'eau et la terre, sont sortis de l'oasis. Les nouvelles lois et opportunités offertes par l'État diminuaient ainsi les marges de manœuvres des propriétaires de droits d'eau des foggaras, qui dépendaient d'une maind'œuvre bon marché. L'État a, en contrepartie, engagé de grandes opérations de réhabilitation et d'aménagement des foggaras. La Direction des ressources en eau a supervisé, par exemple, pour la période 2007-2014, la réhabilitation de 162 foggaras pour un montant équivalent à 13 millions d'euros. Ces opérations étaient souvent en décalage avec la réalité du terrain pour deux raisons. En premier lieu, du fait de l'abaissement du niveau de la nappe, qui diminue chaque année le nombre des foggaras actives. En second lieu, du fait de la raréfaction de la main-d'œuvre qualifiée pour l'entretien des foggaras. En définitive, dans le contexte d'un développement des nouveaux périmètres, si des adaptations et des améliorations des conditions sociales et techniques pour les irrigants n'étaient pas réalisées, le maintien du système, dans sa configuration ancienne, semblait être une tâche difficile.

\subsection{D'une cause de déclin à une solution adoptée: réclamer le forage pour renforcer les foggaras}

Pour les trois personnes ressources, les $2 \mathrm{~km}$ qui séparent le périmètre de mise en valeur agricole de leurs foggaras étaient suffisants pour limiter l'impact des pompages des forages sur les foggaras. En revanche, les habitants ont remarqué l'accélération du rabattement de la nappe et la diminution des débits des foggaras juste après l'exploitation d'un forage d'alimentation en eau potable à destination de plusieurs oasis de la commune, en 1992. Ils s'appuient sur l'augmentation notable des débits de certaines foggaras lorsqu'une panne dans la pompe du forage est survenue durant la première année de sa mise en service. Ce constat a conduit les habitants de l'oasis à acheminer gratuitement l'eau potable pour irriguer leurs parcelles et à fermer les vannes qui alimentaient les autres oasis. Un interviewé justifie cette réaction en affirmant que 
" cette eau appartient seulement à ceux qui ont été affectés par le forage». Cette stratégie fut appliquée malgré un total désaccord des responsables locaux qui devaient subvenir aux besoins en eau potable de la population dans les différentes oasis alimentées par le forage.

Dans ce climat de tension, et profitant de la visite en 1992 $\mathrm{du}$ chef du gouvernement algérien, les habitants de Lahmeur ont manifesté sur la route nationale qui passe à côté de l'oasis. Celui-ci a alors chargé un comité de négocier une solution avec les représentants des manifestants qui réclamaient une amélioration de la situation de leurs foggaras. La solution adoptée fut l'installation d'un forage de «renforcement», en référence au programme officiel de réhabilitation des foggaras, ce qui a mis fin aux "années de vaches maigres » selon les termes du président de l'association des foggaras. Ce forage alimente encore l'oasis de Lahmeur, 25 ans après sa mise en service.

\subsection{Des foggaras "hybrides ": intégration du forage, ajustements et continuités dans un nouveau système sociotechnique oasien}

L'introduction du forage et l'alimentation des foggaras par une nouvelle ressource en eau ont été accompagnées de nombreux changements dans l'infrastructure d'irrigation et dans le fonctionnement de l'action collective et des institutions pour faire fonctionner les nouvelles foggaras, qu'on pourra qualifier «d'hybrides». L'eau pompée du forage dans le canal principal est répartie à l'entrée de l'oasis en canaux secondaires vers les cinq foggaras ainsi renforcées. Les irrigants ont mobilisé plusieurs registres pour opérer ce changement et répondre aux nouveaux besoins et aux charges de fonctionnement: le traditionnel et le moderne, le social et l'économique, etc. Des négociations ont commencé une fois le projet d'installation du forage par l'État acquis. Alors que ce forage était destiné à renforcer seulement deux foggaras de l'oasis, la communauté a décidé, pour exprimer sa solidarité, de répartir l'eau du forage entre les cinq foggaras de l'oasis de Lahmeur ayant subi l'effet des rabattements de nappe depuis 1989.

Le changement le plus fondamental a sans doute concerné la redéfinition des droits d'eau entre les protagonistes des différentes classes sociales. Le phénomène d'ascension sociale des anciens khammès a été largement observé dans la région, mais a souvent été décrit comme un processus lent et progressif pour que ceux-ci puissent accéder à la terre, puis à l'eau (Marouf, 2017). Dans notre cas, la redéfinition des droits d'eau, qui s'est opérée de façon accélérée, visait l'attribution d'une partie des droits d'eau aux anciens khammès qui ne possédaient pas de part d'eau dans l'ancien système de partage des ressources en eau. Ceux-ci avaient participé à la manifestation de 1992 et ont ainsi obtenu un droit d'entrée au statut de propriétaire de l'eau. Pour légitimer cette appropriation, la communauté a fait appel au traditionnel maître de l'eau (kial el-ma en arabe). Chaque propriétaire a estimé ses besoins en eau. Après le jaugeage du débit du forage, le maître de l'eau a jugé que la demande était supérieure au débit réel. Cette contrainte a été réglée par l'application des anciennes règles de la foggara, qui stipulent que toute modification naturelle des débits d'eau doit être partagée entre l'ensemble des propriétaires en quantités proportionnelles à leurs parts de départ. L'association a aussi introduit des règles pour permettre la réallocation des droits d'eau en cas de besoin. Les propriétaires qui éprouvent des difficultés à répondre aux charges de gestion du forage, ou qui veulent même abandonner leurs parcelles, ont la possibilité de diminuer leur part au profit d'un autre propriétaire qui voudrait augmenter la sienne ou d'un nouveau propriétaire qui voudrait accéder à l'eau du forage. Dans ce cas, l'association des foggaras récupère la part d'eau pour la remettre à un autre agriculteur selon l'ordre chronologique des demandes faites à l'association. Dans la foggara d'Abbou, par exemple, les documents de l'association montrent que l'on dénombrait 27 propriétaires de droits d'eau en 2004, alors que nos observations de terrain montrent l'existence de 37 parts en 2017. La communauté a aussi maintenu l'utilisation du bassin doté d'ouvertures proportionnelles aux parts des propriétaires (le peigne ou kesria). La motivation exprimée par les personnes ressources était de rendre visible le partage de l'eau afin d'éviter les conflits en cas de changement des parts d'eau.

L'autre changement fondamental concernait l'accès à la terre. Pour les nouveaux propriétaires de parts d'eau qui n'avaient pas de parcelles à exploiter, un nouveau mode de faire-valoir a été adopté, plus avantageux que celui qui s'appliquait pour les anciens khammès, où ceux-ci ne recevaient que le cinquième de la récolte. Les nouveaux contrats concernent souvent des terrains appartenant à la zawiya (école coranique, faisant office d'autorité culturelle et religieuse locale) ou à la mosquée (biens habûs ou wakf), mais parfois aussi des parcelles abandonnées par leurs propriétaires pour diverses raisons. Le kharrâs (fermier et non plus le khammès-métayer, cf. Marouf, 2017) assure l'entretien des palmiers ou leur plantation dans les nouvelles parcelles. Le propriétaire de la terre récupère la récolte de dattes, tandis que le reste des cultures revient au kharrâs, qui paye les charges de la part d'eau qui lui appartient. Les anciens propriétaires de l'eau et de la terre (chorfa, mrabtine - noblesse religieuse et arabe) privilégiaient traditionnellement de garder les parcelles les plus proches du ksar (l'ancien village) et allouaient le reste dans le cadre de contrats de métayage. En comparant le nombre de propriétaires, issus de la classe sociale des anciens khammès, dans chaque foggara par rapport au nombre total de propriétaires, nous avons pu vérifier que cela est toujours le cas aujourd'hui. Ainsi, en 2004, les anciens khammès représentaient 7 des 27 propriétaires (26\%) dans la foggara Abbou qui traverse l'ancien village, 20 des 33 propriétaires (61\%) dans la foggara de Takarraft qui est juste à côté de la première, 25 des 36 propriétaires $(69 \%)$ dans la foggara d'Ait Waman, 24 des 31 propriétaires $(77 \%)$ dans la foggara d'Amrane et 17 des 20 propriétaires ( $85 \%$ ) dans la foggara de Sebkha, qui irrigue les parcelles les plus éloignées du village.

L'hybridation des infrastructures de captage et de partage, ainsi que celle des institutions, se sont conjuguées à une hybridation organisationnelle (Fig. 2). Une association agréée par l'État a été mise en place en 1998, six ans après la première exploitation du forage. Proposée par l'administration locale, son objectif était l'adaptation des institutions informelles et communautaires aux organisations formelles de l'État, dans l'objectif de faciliter les différentes démarches administratives et d'éviter les conflits de gestion du forage. Son bureau a été 


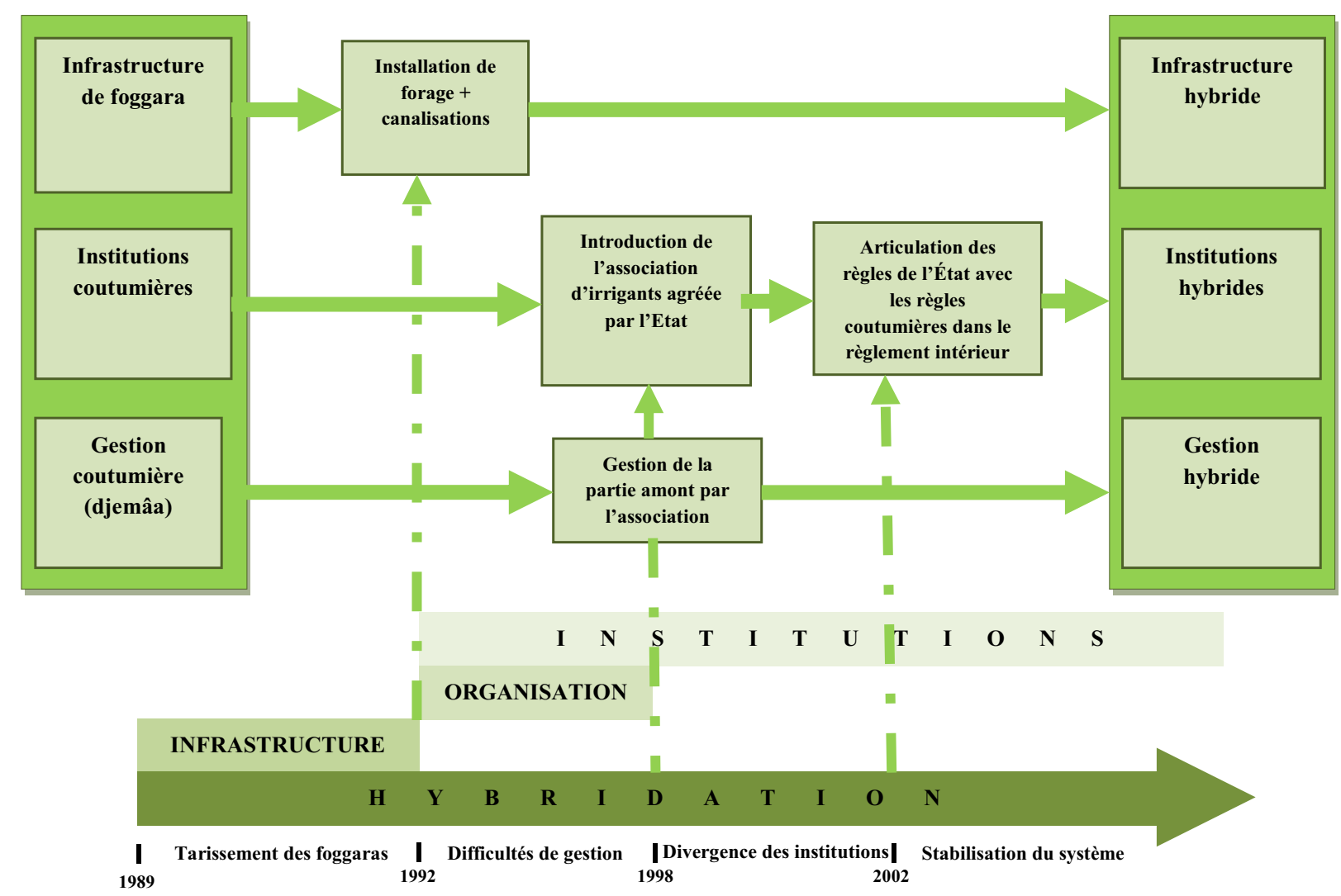

Fig. 2. Processus d'hybridation des foggaras de l'oasis de Lahmeur.

Fig. 2. Hybridization process of foggaras in the Lahmeur oasis.

constitué de représentants des cinq foggaras. Il convient de noter que, durant les quatre premières années qui ont suivi la mise en place de l'association, des problèmes de gestion étaient récurrents. Les habitants, habitués à gérer chaque foggara à part, trouvaient des difficultés à intégrer la nouvelle organisation nécessaire pour gérer le forage (entretien, partage des frais d'énergie, etc.). Pour cela, un dernier ajustement a eu lieu en 2002, par la mise en place d'un règlement intérieur de gestion du forage. Ce dernier, comme indiqué dans sa préface, "...vient sur la demande de l'assemblée générale de l'association des foggaras de Lahmeur après les multitudes de problèmes rencontrés dans la gestion du forage... ». Il décrit de manière détaillée la gestion des conflits, l'attribution des droits d'eau et la gestion du forage. Le forage fut considéré dans le règlement intérieur mis en place comme propriété de l'État, avec une possibilité d'accès pour tous les habitants de l'oasis, sans distinction.

L'ensemble constitué du forage, des canalisations principales, des bassins de distribution principaux et secondaires, et des seguias, a été réparti en deux sous-ensembles (Fig. 3). En amont, le forage et les canalisations principales jusqu'aux cinq bassins principaux, sont sous l'autorité de l'association des foggaras. Cette dernière s'occupe de la gestion du forage et veille à ce que les travaux d'entretien et la récupération des charges soient faits soigneusement. Elle assure le contrôle et le bon partage de l'eau entre les cinq foggaras et fait des ajustements en cas de changement dans le débit du forage. La contribution des propriétaires aux charges de gestion du forage est proportionnelle à leurs parts d'eau. Les irrigants ont souligné un rabattement de la nappe qui continue et qui occasionne des frais d'énergie toujours plus élevés. En aval, chaque bassin de partage principal, ses bassins secondaires et le réseau de distribution secondaire sont gérés par les groupes de propriétaires d'une manière coutumière.

L'analyse des documents et des données de terrain a révélé une recomposition sociale autour des foggaras de Lahmeur. Les membres de la tribu qui dominent aujourd'hui l'association étaient, dans l'ancien système social, classés dans la catégorie des métayers ou étaient considérés comme tels par les autres habitants de l'oasis. Ces personnes ne le reconnaissent toutefois pas, affirmant que leurs origines sont arabes, et qu'ils sont installés dans la zone depuis des siècles, comme en témoigne selon eux leur nom (Draoui) dérivé de l'appellation d'une zone du Maroc (Drâa). Par leur engagement au service de la gestion du forage et les différentes initiatives volontaires qu'ils organisent, ils ont conquis une nouvelle position et assuré leur «ascension sociale». Cela se traduit dans le nombre et l'importance des parts d'eau de ces personnes, qui possédaient 23 des 140 parts d'eau en 2004, l'année durant laquelle ont été mises à jour les listes des propriétaires dans les cinq foggaras, contre moins de 10 parts d'eau avant l'installation du forage.

À l'exception de quelques adaptations, les anciennes pratiques agricoles semblent peu évoluer. Dans les 47 parcelles visitées, le maraîchage, les céréales et les fourrages sont cultivés sous le palmier dattier (deux étages), et parfois sous des arbres fruitiers ou du henné (trois étages). L'association des cultures reste une pratique très répandue et justifiée par le 


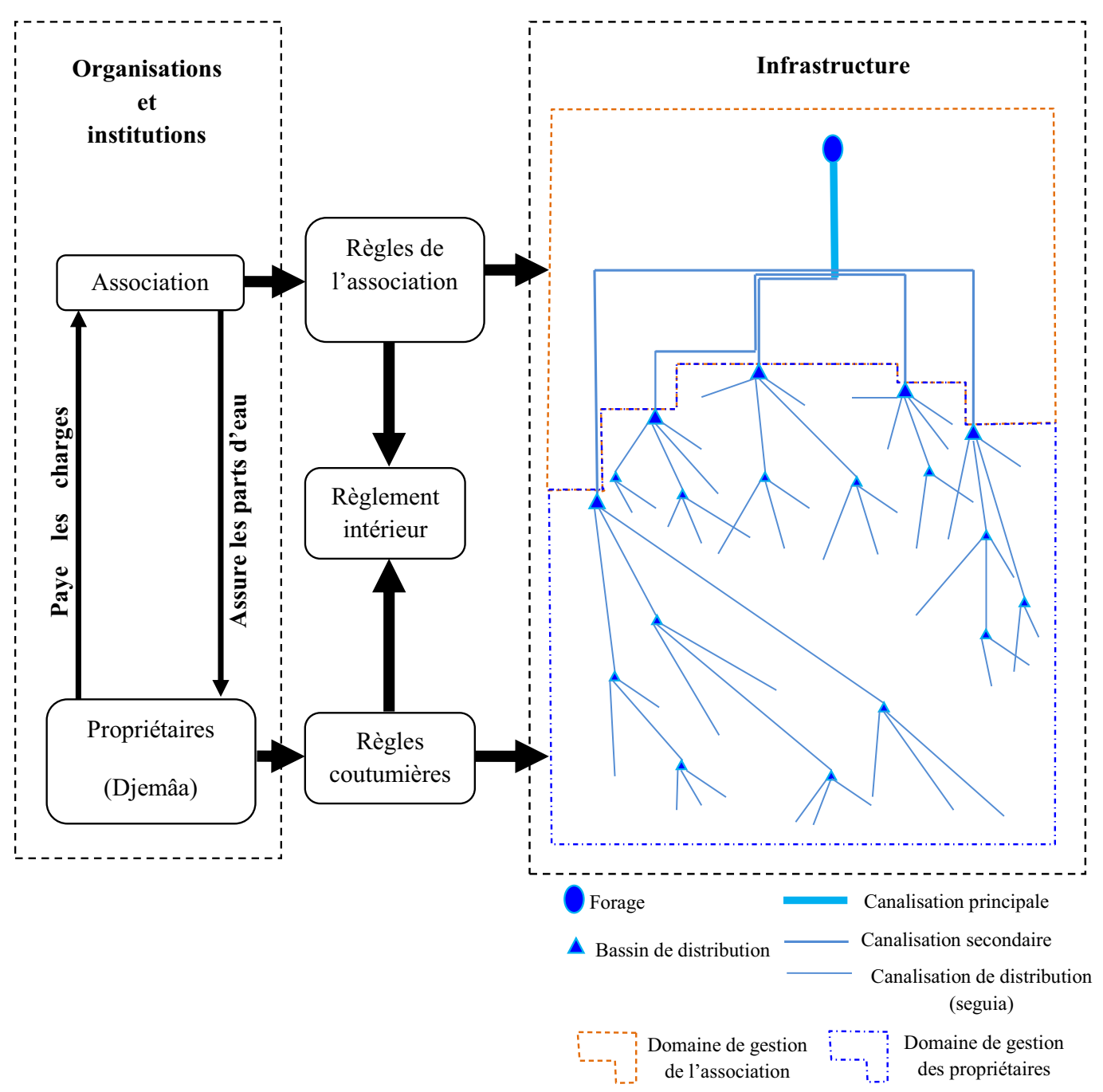

Fig. 3. Schéma synoptique des foggaras hybrides : organisation, institutions et gestion.

Fig. 3. Synoptic diagram of hybrid foggaras: organization, institutions and management.

besoin d'avoir une diversité de produits sur des jardins de surface limitée ainsi que pour créer des conditions d'ombrage favorables pour certaines cultures, particulièrement en été. La plus grande partie des produits est destinée à l'autoconsommation et le reste aux marchés locaux, à l'exception des dattes et du tabac. La culture du tabac s'étend dans les oasis avoisinantes en raison de l'augmentation de la demande à destination des pays du Sud, le Mali et le Niger en particulier. Son prix est attractif, à plus de $7 € / \mathrm{kg}$, contre moins de $1 € / \mathrm{kg}$ pour les dattes. Les 15 enquêtés disent être autosuffisants en produits agricoles, sauf pour les céréales, sur la période allant de janvier à mai ; pour le reste de l'année, une partie importante de l'alimentation provient du marché. Les conditions techniques de travail n'ont pas changé, l'irrigation se fait par submersion et le sol est régulièrement enrichi par des apports de fumier ( 5 à 13 têtes d'ovins et caprins par exploitation). Outre cette activité agricole, qui est souvent une charge attribuée aux personnes âgées et aux femmes, peu concernées par le travail salarial, les membres des familles ont souvent d'autres activités professionnelles. Les jeunes sont attirés par le travail dans les sociétés pétrolières, qui leur assurent de bons salaires, et/ou dans les services de sécurité qui offrent, même avec un niveau d'éducation modeste, des postes de travail permanents. Dans les 15 familles, nous avons recensé 38 jeunes dont 32 fonctionnaires surtout actifs dans les sociétés pétrolières (11) et dans les services de sécurité (7). Une partie de leurs revenus est consacrée à l'amélioration des parcelles et au paiement des charges du forage, qu'ils considèrent indispensable au maintien de leur activité agricole.

\section{Discussion et conclusion}

À travers l'analyse des évolutions qui ont marqué le système des foggaras de Lahmeur, une nouvelle interprétation peut être formulée sur les changements opérés dans les oasis de Touat, Gourara et Tidikelt. Ce qui est qualifié jusqu'à présent dans la littérature (Bellal et al., 2016; Bendjelid et al., 1999; Remini et Achour, 2016), et même dans une bonne partie des discours au niveau local, comme rupture, déclin, bouleversement et fragilisation de l'équilibre oasien ancestral, masque parfois l'émergence de nouveaux modes de fonctionnement affectant les anciennes oasis. Les modifications apportées sont 
des adaptations avec de nombreuses continuités dans les savoirs et les expériences hérités du système oasien séculaire.

L'étude de cas de l'oasis de Lahmeur montre l'existence d'une nouvelle dynamique dans la gestion des foggaras, confirmant ainsi l'hypothèse de Bisson (1992). Paradoxalement, les fortes baisses de la nappe, le déclin des foggaras et la disponibilité des moyens modernes d'exhaure, ont conduit les paysanneries à inventer de nouvelles foggaras. Par les ajustements apportés, celles-ci ont montré une forte capacité d'adaptation à l'environnement et au nouveau contexte socioéconomique. La communauté a d'abord opéré des changements dans les droits d'accès à l'eau et à la terre afin d'éviter le départ des anciens khammès, agriculteurs dynamiques détenteurs de savoirs. Ensuite, des ajustements ont été apportés à l'infrastructure hydraulique et aux institutions qui gouvernent la gestion de l'eau. L'hybridation des eaux par le forage permet un nouveau mode d'alimentation dans la zone, pour faire face au rabattement de la nappe. Par une contribution aux charges de gestion des membres de l'association, le forage a permis de faire face au manque d'entretien des foggaras de la zone. Dans d'autres oasis visitées, les ajustements étaient sensiblement différents. À $10 \mathrm{~km}$ de Lahmeur, dans l'oasis de Tittaf par exemple, un forage de renforcement est exploité en parallèle de la foggara, par un réseau de distribution indépendant. Dans ce cas, la remise à plat de l'ensemble des droits d'eau n'a pas eu lieu et les propriétaires de la foggara se sont opposés à son renforcement par le forage. Le choix d'un réseau parallèle était une adaptation à des contraintes spécifiques à l'oasis. La première raison fut la topographie qui ne permettait pas, en cas d'intégration du forage à la foggara, l'irrigation des parcelles des nouveaux propriétaires en amont. La deuxième raison était que la foggara apportait encore des volumes d'eau non négligeables et que les propriétaires des grandes parts d'eau de la foggara n'acceptaient pas de redéfinir les parts d'eau.

Le cas d'étude met en avant certaines pratiques héritées de l'ancien système. L'action collective reste encore au centre de la dynamique de l'oasis comme dans d'autres systèmes de gestion des ressources communes, en particulier dans les environnements hostiles. Le fonctionnement actuel plus ou moins stable des cinq foggaras hybrides résulte d'une évolution emblématique dans l'organisation et les règles de gestion du système. A travers un mélange savant de gestion coutumière et «moderne» de l'infrastructure hybride, les membres du groupe ont façonné les institutions. Ils ont créé le chaînon manquant en adoptant un règlement intérieur dont le contenu a été élaboré pour gérer cette infrastructure hybride. L'organisation mise en place reproduit l'un des principes de conception de la gestion des communs proposés par Ostrom (1990). Il s'agit de l'imbrication du système en couches horizontales et verticales ; soit, dans notre cas d'étude, entre les cinq foggaras, et entre l'ensemble des foggaras et l'association.

À la lumière de notre étude, la problématique de durabilité des systèmes de foggaras peut se résumer aux deux composantes suivantes :

- la première est l'importance à accorder à la réflexion et à l'action dans les programmes de développement, pour accompagner l'évolution des foggaras en parallèle avec les transformations sociales, économiques et environnementales, et ce en coordination avec les oasiens. Les foggaras sont encore vues comme des systèmes non évolutifs qu'il s'agit de réhabiliter. Une nouvelle vision de ce que peut être une foggara aujourd'hui, i.e. un système bien vivant, serait nécessaire pour réussir ces programmes de développement (Bisson, 1992);

- la deuxième est la capacité des oasiens à s'adapter aux transformations dans leur environnement physique et socioéconomique. Cette capacité sera confrontée aux inégalités d'accès à l'eau souterraine par rapport aux périmètres de mise en valeur agricole, et à la désaffection des jeunes vis-à-vis du travail agricole, car ils sont de plus en plus séduits par les activités salariales en dehors des ksour (pluriel de ksar) et des oasis traditionnelles.

\section{Références}

Balland D. 1992. Les eaux cachées: études géographiques sur les galeries drainantes souterraines. Publications du Département de Géographie de l'Université de Paris-Sorbonne n ${ }^{\circ} 19$.

Bellal SA, Hadeid M, Ghodbani T, Dari O. 2016. Accès à l'eau souterraine et transformations de l'espace oasien: le cas d'Adrar (Sahara du Sud-ouest algérien). Cahiers de géographie du Québec 60(169): 29-56. DOI: 10.7202/1038664ar.

Bendjelid A, Dari O, Hadeid M, Bellal SA, Gacem F, Belmahi MN, et al. 1999. Mutations sociales et adaptation d'une paysannerie ksourienne du Touat: Ouled Hadj Mamoun (wilaya d'Adrar, Algérie). Insaniyat 7: 39-52. DOI: 10.4000/insaniyat.12147.

Bisson J. 1992. Les foggaras du Sahara algérien : déclin ou renouveau. In: Balland D, ed. Les eaux cachées. Études géographiques sur les galeries drainantes souterraines, pp. 7-26.

Bisson J. 2003. Le Sahara: mythes et réalités d'un désert convoité. Paris: L'Harmattan, 479 p.

Côte M. 2002. Des oasis aux zones de mise en valeur: l'étonnant renouveau de l'agriculture saharienne. Méditerranée 99(3): 5-14. DOI: 10.3406/medit.2002.3253.

Daoudi A, Colin J-P, Derderi A, Ouendeno ML. 2015. Mise en valeur agricole et accès à la propriété foncière en steppe et au Sahara (Algérie). Les Cahiers du Pôle Foncier 13: 34.

Dubost D, Moguedet G. 1998. Un patrimoine menacé : les foggaras $\mathrm{du}$ Touat. Science et changements planétaires/Sécheresse 9(2): $117-122$

El Faiz M, Ruf T. 2010. An Introduction to the Khettara in Morocco: Two Contrasting Cases. In: Schneier-Madanes G, Courel M-F, ed. Water and Sustainability in Arid Regions: Bridging the Gap Between Physical and Social Sciences. Dordrecht: Springer (Netherlands), pp. 151-163.

Lightfoot DR. 1996. Moroccan khettara: Traditional irrigation and progressive desiccation. Geoforum 27(2): 261-273. DOI: 10.1016/ 0016-7185(96)00008-5.

Marouf N. 2017. Droits d'eau, hiérarchies en mouvement au Touat et Gourara: Radioscopie d'une société hydraulique. In: Guignard D, ed. Propriété et société en Algérie contemporaine. Quelles approches? Aix-en-Provence: IREMAM. DOI: 10.4000/books. iremam.3717.

Mostafaeipour A. 2010. Historical background, productivity and technical issues of Qanats. Water History 2(1): 61-80. DOI: 10.1007/s12685-010-0018-z.

Ostrom E. 1990. Governing the commons: the evolution of institutions for collective action. Political economy of institutions and decisions. Cambridge: Cambridge University Press, $298 \mathrm{p}$.

Otmane T, Kouzmine T. 2013. Bilan spatialisé de la mise en valeur agricole au Sahara algérien. Cybergeo: European Journal of 
Geography 632. Disponible sur: http://cybergeo.revues.org/25732. DOI: $10.4000 /$ cybergeo.25732.

Papy L. 1959. Le déclin des foggaras au Sahara, d'après des travaux récents. Cahiers d'outre-mer 48: 401-406.

Remini B, Achour B. 2016. The water supply of oasis by albian foggara: an irrigation system in degradation. Larhyss Journal 26: 167-181.
Sahli Z. 1997. Deux tentatives controversées de modernisation de l'agriculture en zone aride : l'opération 'tomate d'Adrar' et la mise en valeur hydro-agricole du Touat Gourara (Wilaya d'Adrar Algérie). In: Jouve AM, ed. La modernisation des agricultures méditerranéennes (à la mémoire de Pierre Coulomb). Ciheam (Montpellier): Options Méditerranéenne, série A 29, pp. 283-295.

Citation de l'article : Idda S, Bonté B, Mansour H, Bellal S-A, Kuper M. 2017. Monument historique ou système bien vivant? Les foggaras des oasis du Touat (Algérie) et leur réalimentation en eau par pompage. Cah. Agric. 26: 55007. 